Check for updates

Cite this: Phys. Chem. Chem. Phys., 2017, 19, 18777

\section{Correction: Structural characterization of gas-phase cysteine and cysteine methyl ester complexes with zinc and cadmium dications by infrared multiple photon dissociation spectroscopy}

\author{
Rebecca A. Coates, ${ }^{a}$ Christopher P. McNary, ${ }^{a}$ Georgia C. Boles, ${ }^{a}$ Giel Berden, ${ }^{b}$ \\ Jos Oomens ${ }^{\text {bc }}$ and P. B. Armentrout (D) *a
}

DOI: $10.1039 / \mathrm{c} 7 \mathrm{cp} 90144 \mathrm{e}$

Correction for 'Structural characterization of gas-phase cysteine and cysteine methyl ester complexes with zinc and cadmium dications by infrared multiple photon dissociation spectroscopy' by Rebecca A.

rsc.li/pccp

Although the overall conclusions of the original article remain unaffected (no experimental or theoretical IR spectra are changed, nor is any calculated thermochemistry at $0 \mathrm{~K}$ ), the thermal corrections to the Gibbs free energy at $298 \mathrm{~K}$ were mistakenly overestimated. Corrected $298 \mathrm{~K}$ values for Tables 1 and 2 from the original manuscript are given below. Notably, only very subtle changes are found such that the relative order of all $298 \mathrm{~K}$ theoretically determined low-energy species remains the same for the $[\mathrm{Zn}(\mathrm{Cys}-\mathrm{H})]^{+},[\mathrm{Cd}(\mathrm{Cys}-\mathrm{H})]^{+},[\mathrm{Zn}(\mathrm{CysOMe}-\mathrm{H})]^{+},[\mathrm{Cd}(\mathrm{CysOMe}-\mathrm{H})]^{+}$and $\mathrm{CdCl}^{+}(\mathrm{CysOMe})$ systems. Therefore, we have not corrected the relative energies used throughout the text.

Table 1 B3LYP, ${ }^{a}$ B3P86, and MP2(full) relative free energies $\left(\mathrm{kJ} \mathrm{mol}^{-1}\right)$ at $298 \mathrm{~K}$ of low-lying conformers of $[\mathrm{M}(\mathrm{Cys}-\mathrm{H})]^{+}$and $[\mathrm{M}(\mathrm{Cys} \mathrm{OMe}-\mathrm{H})]^{+b}$

\begin{tabular}{|c|c|c|c|}
\hline Complex & Structure & $\mathrm{Zn}$ & $\mathrm{Cd}$ \\
\hline$[\mathrm{M}(\mathrm{CysOMe}-\mathrm{H})]^{+}$ & $\begin{array}{l}{\left[\mathrm{N}, \mathrm{CO}, \mathrm{S}^{-}\right] \operatorname{tgg}} \\
{\left[\mathrm{N}, \mathrm{CO}, \mathrm{S}^{-}\right] \operatorname{cgg}^{-}} \\
{\left[\mathrm{N}, \mathrm{OMe}, \mathrm{S}^{-}\right] \operatorname{tgg}} \\
{\left[\mathrm{N}, \mathrm{S}^{-}\right] \operatorname{tgt}} \\
{\left[\mathrm{CO}, \mathrm{S}^{-}\right] \operatorname{tgg}} \\
{\left[\mathrm{CO}, \mathrm{S}^{-}\right] \operatorname{ctg}} \\
{\left[\mathrm{N}, \mathrm{S}^{-}\right] \operatorname{cgt}} \\
{\left[\mathrm{N}^{-}, \mathrm{CO}, \mathrm{S}\right] \operatorname{tggg}}\end{array}$ & $\begin{array}{l}0.0(0.0), 0.0,0.0 \\
36.2(33.4), 35.2,36.2 \\
43.7(38.9), 46.3,37.2 \\
36.9(41.2), 43.4,46.5 \\
55.5(61.2), 64.0,74.1 \\
76.9(77.6), 83.5,93.6 \\
84.3(84.7), 89.5,92.8 \\
102.3(102.6), 103.3,105.9\end{array}$ & $\begin{array}{l}0.0(0.0), 0.0,0.0 \\
37.2(34.6), 36.0,37.6 \\
36.7(32.8), 39.5,33.2 \\
27.0(32.4), 31.5,37.2 \\
50.7(57.4), 57.3,70.0 \\
72.3(74.2), 77.0,90.6 \\
73.1(74.5), 76.3,82.1 \\
104.4(104.6), 106.4,112.7\end{array}$ \\
\hline
\end{tabular}

${ }^{a}$ Values including empirical dispersion corrections are given in parentheses. ${ }^{b}$ Calculations performed at the B3LYP, B3P86, and MP2(full) levels of theory using a 6-311+G(2d,2p) basis set for Zn-containing complexes and def2TZVPP for Cd-containing complexes. Geometries and vibrational frequencies calculated at the B3LYP/6-311+G(d,p) level for Zn-containing complexes and B3LYP/def2TZVP for Cd-containing complexes. ${ }^{c}$ Salt bridge between $\mathrm{NH}^{+}, \mathrm{CO}_{2}^{-}$, and $\mathrm{S}^{-}$groups.

\footnotetext{
${ }^{a}$ Department of Chemistry, University of Utah, Salt Lake City, UT 84112, USA. E-mail: armentrout@chem.utah.edu

${ }^{b}$ Radboud University, Institute for Molecules and Materials, FELIX Laboratory, Toernooiveld 7, NL-6525ED Nijmegen, The Netherlands

'van't Hoff Institute for Molecular Sciences, University of Amsterdam, Amsterdam, The Netherlands
} 
Table 2 Relative free energies $\left(\mathrm{kJ} \mathrm{mol}^{-1}\right)$ at $298 \mathrm{~K}$ of low-lying $\mathrm{CdCl}^{+}(\mathrm{CysOMe})$ conformers ${ }^{a}$

\begin{tabular}{|c|c|c|c|}
\hline Structure & $\mathrm{B} \mathrm{LYP}^{b}$ & В3Р86 & MP2(full) \\
\hline$[\mathrm{N}, \mathrm{CO}, \mathrm{S}] \operatorname{tggg}_{-}$ & $1.1(1.7)$ & 1.2 & 1.7 \\
\hline $\left.\mathrm{S}^{-}\right] t c g^{x}$ & $17.6(19.0)$ & 18.2 & 21.5 \\
\hline$[\mathrm{N}, \mathrm{CO}]$ tggg & $20.7(29.2)$ & 25.3 & 30.7 \\
\hline$[\mathrm{N}, \mathrm{CO}] \operatorname{tcg} g$ & $21.2(30.2)$ & 25.9 & 31.8 \\
\hline$[\mathrm{N}, \mathrm{S}] \operatorname{tgtg}$ & $23.4(30.3)$ & 26.0 & 33.7 \\
\hline$[\mathrm{N}, \mathrm{OM}, \mathrm{S}] \operatorname{tggg}_{-}$ & $32.2(27.7)$ & 33.4 & 26.7 \\
\hline$[\mathrm{N}, \mathrm{CO}, \mathrm{S}] \operatorname{cggg}$ & $38.8(36.1)$ & 37.9 & 39.2 \\
\hline$[\mathrm{CO}, \mathrm{S}] \mathrm{ttgt}$ & $51.0(58.8)$ & 56.8 & 72.6 \\
\hline$\left[\mathrm{CO}, \mathrm{S}^{-}\right] \operatorname{cgg}^{c}$ & $66.2(66.8)$ & 64.7 & 72.2 \\
\hline$[\mathrm{CO}, \mathrm{S}] \mathrm{ctg} t$ & $82.5(88.9)$ & 87.6 & 104.2 \\
\hline
\end{tabular}

${ }^{a}$ Calculations performed at the stated level of theory using a def2TZVPP basis set. Geometries and vibrational frequencies calculated at the B3LYP/ def2TZVP level of theory. ${ }^{b}$ Values including empirical dispersion corrections are given in parentheses. ${ }^{c}$ Salt bridge between $\mathrm{NH}^{+}$, $\mathrm{CO}_{2}{ }^{-}$, and $\mathrm{S}^{-}$ groups.

The Royal Society of Chemistry apologises for these errors and any consequent inconvenience to authors and readers. 